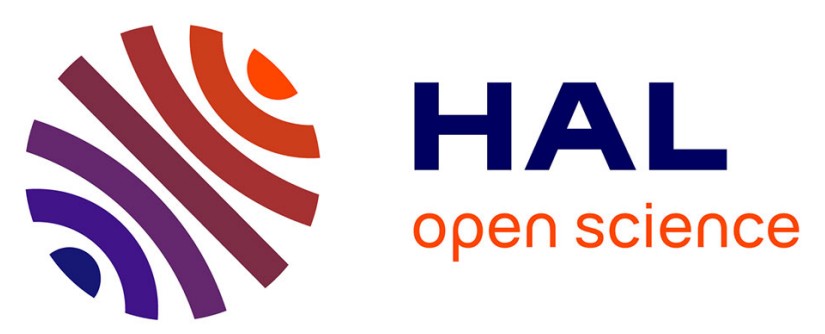

\title{
Selective vulnerability of the primitive meningeal layer to prenatal Smo activation for skull base meningothelial meningioma formation
}

Julien Boetto, Caroline Apra, Matthieu Peyre, Franck Bielle, Michel Kalamarides

\section{To cite this version:}

Julien Boetto, Caroline Apra, Matthieu Peyre, Franck Bielle, Michel Kalamarides. Selective vulnerability of the primitive meningeal layer to prenatal Smo activation for skull base meningothelial meningioma formation. Oncogene, 2018, 37 (36), pp.4955-4963. 10.1038/s41388-018-0328-7 . hal01991334

\section{HAL Id: hal-01991334 \\ https://hal.sorbonne-universite.fr/hal-01991334}

Submitted on 23 Jan 2019

HAL is a multi-disciplinary open access archive for the deposit and dissemination of scientific research documents, whether they are published or not. The documents may come from teaching and research institutions in France or abroad, or from public or private research centers.
L'archive ouverte pluridisciplinaire HAL, est destinée au dépôt et à la diffusion de documents scientifiques de niveau recherche, publiés ou non, émanant des établissements d'enseignement et de recherche français ou étrangers, des laboratoires publics ou privés. 


\title{
Selective vulnerability of the primitive meningeal layer to prenatal Smo activation for skull base meningothelial meningioma formation
}

\author{
Julien Boetto ${ }^{1}$ Caroline Apra ${ }^{1} \cdot$ Franck Bielle $^{1,2} \cdot$ Matthieu Peyre ${ }^{1,3} \cdot$ Michel Kalamarides $^{1,3}$
}

\begin{abstract}
Somatic activating mutations of smoothened (SMO), a component of the embryonic sonic hedgehog (SHH) signaling pathway, are found in 3-5\% of grade I meningiomas, most of them corresponding to meningothelial meningiomas located at the anterior skull base. By generating different developmental stage-specific conditional activations in mice, we define a restricted developmental window during which conditional activation of Smo in Prostaglandin D2-synthase-positive mesoderm-derived meningeal layer of the skull base results in meningothelial meningioma formation. We show a selective vulnerability of the arachnoid from the skull base to Smo activation to initiate tumor development. This prenatal period and specific topography are correlated to the timing and location of SHH signaling involvement in the formation of craniofacial and meninges patterning, strongly corroborating the hypothesis of a developmental origin for Smo-activated meningiomas. Finally, we provide preclinical in vitro evidence of the efficacy of the SMO-inhibitor Sonidegib, supporting further preclinical and clinical evaluation of targeted treatment for refractory $S M O$-mutant meningiomas.
\end{abstract}

\section{Introduction}

Meningiomas are the most common primary central nervous system tumors in adults [1]. Treatment algorithms are based on surveillance, surgery, and radiotherapy/radiosurgery [2]. Meningiomas are classified into three prognostic histological groups following the WHO classification: grade I (65-80\%), grade II (20-35\%, atypical), and grade III ( $<3 \%$, anaplastic) [1,2]. Recent advances in the molecular genetics of WHO grade I meningioma have led to the discovery of mutations in several oncogenes, including $A K T 1, K L F 4$,

Electronic supplementary material The online version of this article (https://doi.org/10.1038/s41388-018-0328-7) contains supplementary material, which is available to authorized users.

Michel Kalamarides

michel.kalamarides@aphp.fr

1 CRICM INSERM U1127 CNRS UMR 7225, Institut du Cerveau et de la Moelle Epinière, Sorbonne Université, Paris F-75013, France

2 Department of Pathology, AP-HP, Hôpital Pitié-Salpêtrière, Paris F-75013, France

3 Department of Neurosurgery, AP-HP, Hôpital Pitié-Salpêtrière, Paris F-75013, France
SMO, PIK3CA, POLRA2, and TRAF7, all mutually exclusive to mutations in the NF2 tumor suppressor gene involved in at least $50 \%$ of meningiomas [3-6]. Discovery of this mutational landscape enabled the establishment of histological and/or anatomical correlations with specific gene mutations $[3,7,8]$. In a recent series of 775 meningiomas, Clark et al. described Sonic Hedgehog (SHH) pathway-related meningiomas harboring $42 S M O$ gene mutations (recurrent mutations L412F and W535L in $76 \%$ of cases), 5 SUFU mutations and 3 PRKARIA mutations [4]. These tumors were mostly WHO grade I, meningothelial, and located at the anterior skull base, suggesting a possible link between this potential driver mutation and anatomical site of origin. Indeed, SMO mutations alter the SHH pathway, which plays a critical role in craniofacial patterning during embryonic development, and consequently in the development of meninges at this location [9]. However, it is not clear whether meningioma tumorigenesis is induced by early prenatal deregulation of meningeal embryogenesis or by late postnatal dedifferentiation of mature cells into a stem-like state [4]. In fact, very little is known about the origin and regulation of meningeal development in humans and mice. By generating different tissue and developmental stage-specific conditional knockout mice with neural crest and mesoderm specific promoters, we and others have characterized the embryonic 
Table 1 Summary of the phenotypic consequences of Smo activation in meningeal cells in vivo

\begin{tabular}{|c|c|c|c|c|}
\hline Mouse genotype Phenotypic abnormality & PGDSCre-SmoM2 $n=42$ & $\begin{array}{l}\text { AdCre-injected SmoM2 } n= \\
53\end{array}$ & $\begin{array}{l}\text { AdLacZ-injected SmoM2 } n= \\
8\end{array}$ & \\
\hline Meningothelial meningioma & $9(21 \%)$ & $1(2 \%)$ & 0 & $p=0.003$ \\
\hline Meningothelial proliferation & $32(76 \%)$ & $21(43 \%)$ & 0 & $p<0.001$ \\
\hline Medulloblastoma & 0 & $4(8 \%)$ & 0 & $p=0.14$ \\
\hline
\end{tabular}

origin of the meninges with those covering the telencephalon being of neural crest origin, and those at the level of the skull base deriving from the cephalic mesoderm [1012]. We showed that prostaglandin D2 synthase (PGDS) gene is a specific marker of arachnoidal cells and meningiomas in humans and mice and that PGDS-positive cells of the primitive meningeal layer developed during embryogenesis are the progenitor cells of origin capable of generating meningiomas [12]. Here we demonstrate that mouse meningiomas induced by Smo activation in PGDS-positive cells have the same histological-meningothelial-and anatomical-skull base-characteristics as their human $S M O$-mutant tumors counterparts. In addition, we have identified a specific temporal window of activation for Smorelated meningioma tumorigenesis, providing clues to the developmental origin of this subgroup of meningiomas. These findings provide new insights in the molecular mechanisms of meningioma formation and support the use of targeted medical therapy for those rare skull base SMOmutant meningiomas that are refractory to surgery and radiotherapy/radiosurgery with high morbidity [13].

\section{Results}

\section{Identification of a restricted developmental temporal window for Smo activation and meningioma vulnerability}

To investigate the role of Smo mutation in meningeal tumorigenesis, we generated genetically engineered SmoM2 mice in which activation of the Shh pathway was achieved by Cre-mediated conditional expression of an activated Smoothened allele (SmoM2) [14]. Prenatal activation of Smo was induced by generating 42 PGDSCre;SmoM2 mice where SmoM2 was expressed early (starting at E12.5) at the skull base level in the meningeal precursor cells identified as PGDS-positive cells [12]. After a mean survival of 14.2 months, 9 of 42 (21\%) PGDSCre;SmoM2 mice developed meningiomas and $32(76 \%)$ presented foci of meningothelial proliferation, the first manifestation of meningioma tumorigenesis in mice [15] (Table 1). All tumor lesions were localized at the skull base level ventral to the brainstem. Histologically, they appeared as meningothelial grade I meningiomas showing the typical aspect of tumoral meningothelial cells with oval nuclei and nuclear clearing disposed in lobules (Fig. 1a-c).

To analyze the effect of postnatal Smo activation, a cohort of 53 SmoM2 mice was injected with adCre at postnatal day 2 (25 at the convexity-s.d. (subdural)- and 28 at the skull base-t.o.(trans orbitary)-). Forty-nine mice were analyzed after a mean survival of 14.2 months: only one meningothelial meningioma (2\%) and 23 (43\%) meningothelial proliferations were observed at the skull base (Fig. 1d). All meningothelial proliferations were located at the skull base level even after injection at the convexity. Interestingly, the four other mice in the cohort died shortly after a mean survival of 2.8 months. All presented typical large cerebellar medulloblastomas composed by sheets of poorly differentiated small cells (Fig. 1e), without meningothelial proliferation. Our hypothesis is that after sub-dural injection the adCre virus transduced cells in the neuraxis by diffusion through the cerebrospinal fluid. This induced SmoM2 expression in neural progenitors of developing cerebellar cortex, the cell of origin of Shh-activated medulloblastoma representing $25 \%$ of this tumor type $[16,17]$. Activation of the Hedgehog pathway was confirmed in vivo in both models by immunohistochemical expression of Gli-1 in Smo-related meningothelial proliferations and meningiomas (Fig. 1f, g). No meningothelial proliferation, meningioma, or medulloblastoma were found in the control cohort of 8 adLacZ-SmoM2 mice. These results defined a restricted developmental window during which conditional activation of Smo in PGDS mesodermderived meningeal layer of the skull base results in meningioma formation.

\section{Selective vulnerability of the skull base compared to convexity arachnoidal cells to SmoM2 expression on proliferation and Shh pathway activation}

To analyze the effect of Smo activation in the meninges according to their location and embryologic origin, we established primary arachnoidal cell cultures from meninges of the skull base or the convexity of SmoM2 mice. After adCre infection of primary arachnoidal cells in vitro, SmoM2 activation was assessed by detection of YFP fluorescence (Fig. 2a, b). No fluorescence was expressed in 
Fig. 1 Pathological

characterization of Smo-induced tumors in PGDSCre;SmoM2 mice and $a d C r e ; S m o M 2$ mice. a H\&E stained sections showing meningothelial meningioma overlying the brain (arrowhead, $\times 200$ ). b Another

meningothelial meningioma composed of typical tumoral arachnoidal cells with oval nuclei at higher magnification ( $\times 400)$. c H\&E stained section showing normal arachnoidal layer $(\mathbf{c}$, arrowhead $\times 200)$ and a meningothelial proliferation (d, arrowhead $\times 200$ ). e H\&E-Safran stained section of a large medulloblastoma (star, $\times 200$ ) invading the cerebellum). Gli-1 immunohistochemistry in a meningothelial proliferations (f) and a meningothelial meningioma (g) demonstrating positivity of meningioma cells. $\mathrm{Br}$ brain, $\mathrm{tg}$ trigeminal nerve, cer Cerebellum
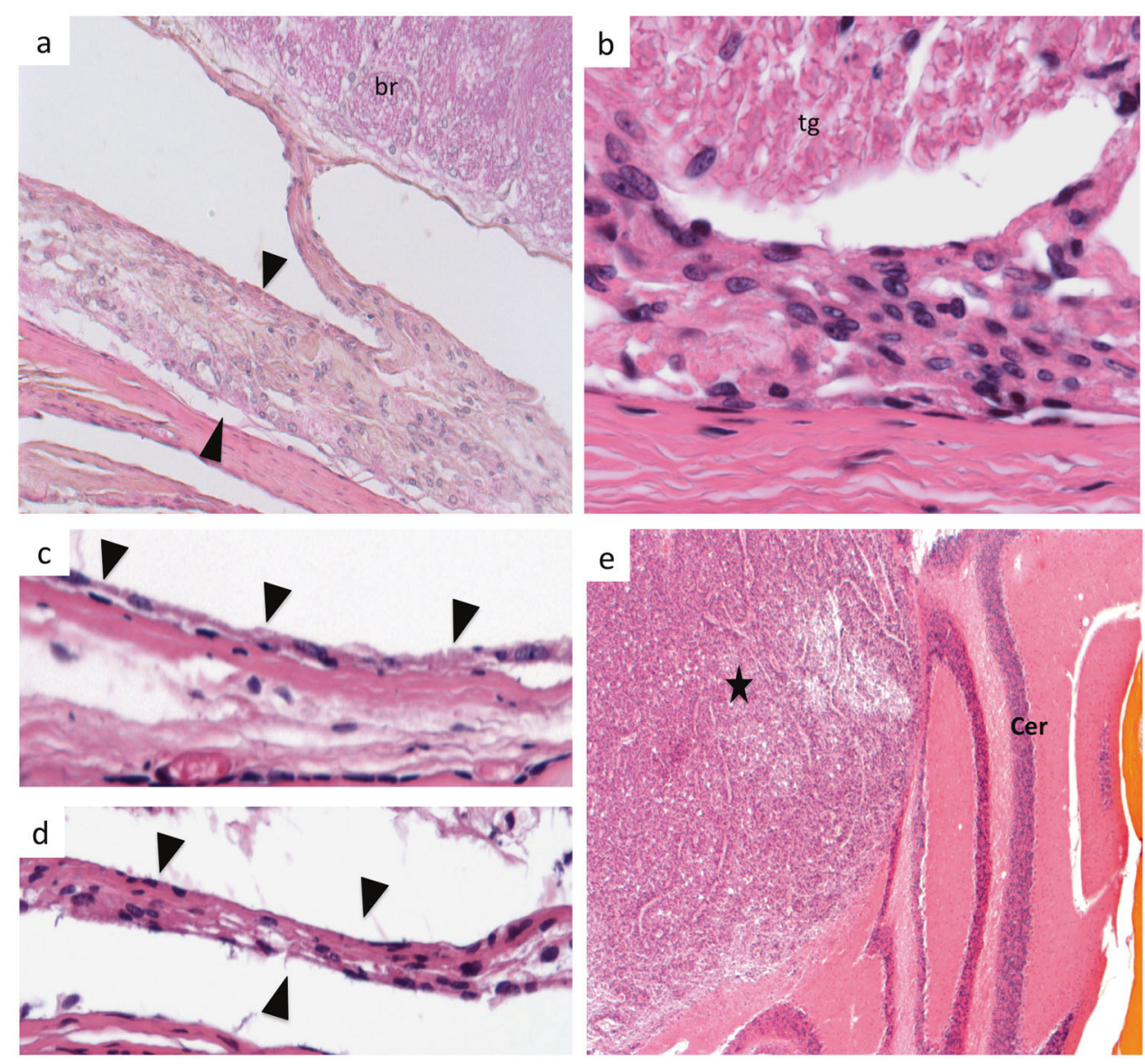

$\mathrm{f}$

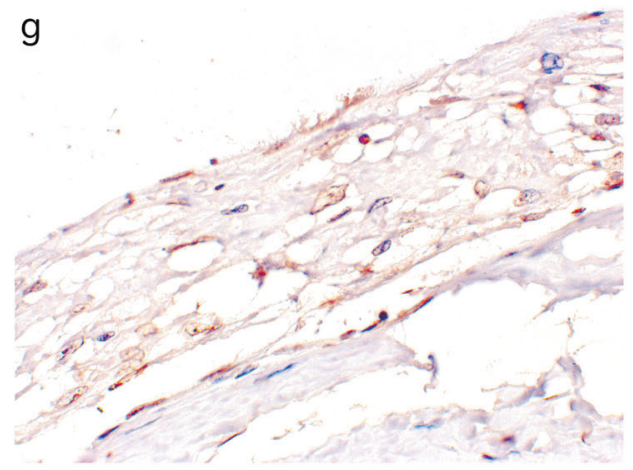

the adLacZ-infected control SmoM2 cells (data not shown). Proliferation assays showed that SmoM2 expression induced a slight proliferative advantage in skull base arachnoidal cells at day 7 ( $p=0.009, t$-test, Fig. $2 c)$, whereas no significant effect was seen in convexity arachnoidal cells ( $p=0.85$, $t$-test, Fig. 2d). WST1 activity was not significantly modified by SmoM2 expression in skull base arachnoidal cells compared to convexity arachnoidal cells ( $p=0.46, t$-test, Fig. 3b). Contrary to expectations, apoptosis was significantly increased when SmoM2 was expressed (16.7 vs $8.1 \%, p<0.001$, Fig. $2 \mathrm{~g}-\mathrm{i})$. On the other hand, cellular senescence was not activated by SmoM2 expression as demonstrated by the SA $\beta$-galactosidase assay (data not shown). In skull base arachnoidal cells, SmoM2 expression induced a significant increase in Glil ( $p=0.005$, $t$-test) and Ptchl ( $p=0.03, t$-test) mRNA levels (Fig. 2e), the two main target genes of the canonical Shh pathway [18]. In contrast, no difference in Glil ( $p=0.37, t$-test) or Ptchl ( $p=0.77, t$-test) mRNA levels was found in the convexity arachnoidal cells (Fig. 2f). The differences in arachnoid from skull base and convexity region are not intrinsic as growth pattern and mRNA levels of Glil and Ptchl were similar in control/wild-type cells (infected with adLacZ). These results clearly showed a differential response and thus vulnerability to Smo activation according to the location and embryological origin of the meninges, 

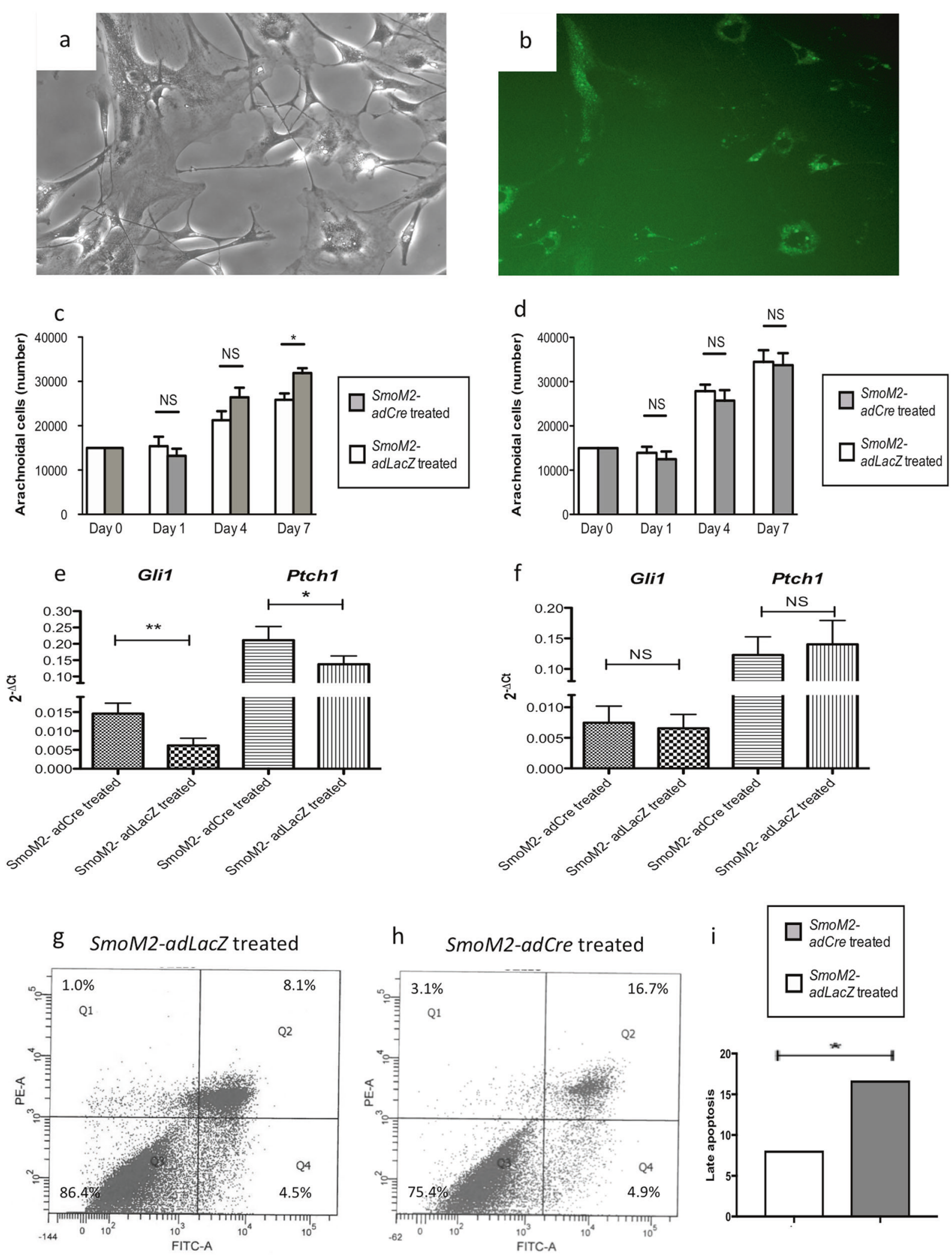
Fig. 2 Proliferation assays and SHH-pathway activation study showing a spatial restriction of Smo activation effect depending on topographic origin of arachnoidal cells. Morphologies of primary skull base SmoM2 arachnoidal cells in culture after adCre infection (a), showing SmoM2 activation assessed by expression of YFP in fluorescence microscopy (b). Proliferation assays on skull base (c) and convexity (d) arachnoidal cells: SmoM2 expression induces a proliferation advantage among skull base arachnoidal cells at day 7 ( $p=$ $0.009, t$-test) but not among convexity cells ( $p=0.85, t$-test). Comparison of Glil and Ptchl mRNA levels among skull base (e) and convexity cells (f): SmoM2 expression induces a significant increase in Glil ( $p=0.005, t$-test) and Ptchl ( $p=0.03, t$-test) among skull base arachnoidal cells but not among convexity cells $(p=0.37$ and $0.77, t$ test). Apoptosis assay: annexin V versus propidium iodide (PI) cell sorting for skull base $S m o M 2$ arachnoidal cells infected with adLacZ virus (g) or adCre virus (h). The percentage of Annexin V-positive/PInegative cells (early stage of apoptosis, Q4) and Annexin V-positive/ PI-positive cells (late stage of apoptosis, Q2) were separated and measured using flow cytometry. Late apoptosis was significantly higher in cells expressing SmoM2 (i) (16.7 versus 8.1\%, $p<0.001)$. All experiments were performed in triplicate

those of the skull base being exquisitely sensitive to Shh pathway activation.

\section{LDE-225 (Sonidegib) inhibits Shh pathway and cell proliferation in skull base SmoM2 arachnoidal cells}

To obtain preclinical evidence of the efficacy of SMO inhibitors in the treatment of meningiomas, we analyzed the effect of LDE-225 (Sonidegib) on primary arachnoidal cells in culture. Since the pro-proliferative effect of SmoM2 expression was found only in arachnoidal cells of the skull base, all the experiments with Sonidegib were carried out with those cells. Skull base adCre-infected SmoM2 arachnoidal cells were treated with Sonidegib (10 or $100 \mathrm{nM})$ 1 day after adCre infection. Short-term proliferation assays showed that Sonidegib at $10 \mathrm{nM}$ was able to inhibit the proliferation of adCre-infected SmoM2 cells in terms of cell count ( $p=0.02, t$-test, Fig. 3a) although WST1 activity was not significantly decreased ( $p=0.41, t$-test, Fig. $3 b)$. There was a significant reduction in Glil $(p=0.005, t$-test $)$ mRNA expression compared to vehicle (DMSO) treatment, but no difference was observed for Ptchl mRNA levels ( $p$ $=0.71, t$-test) (Fig. 3c, d).

\section{Discussion}

Among the nine different histological subtypes of Grade I meningiomas in humans, the unique histological subtypemeningothelial- of $S M O$-mutant meningiomas supports the view that this oncogenic pathway is critical to the arachnoidal meningeal lineage at the origin of this subtype compared to the fibroblastic subtype. Previous embryologic studies have shown that PGDS-positive primordial meningeal cells arising at E12.5 give rise to meningeal cells of both arachnoid and dura-mater layers at E14.5 (Fig. 4) [12]. We previously showed using gentically engineered mouse models that the two major histological subtypes of WHO Grade I meningiomas were inducible by $N f 2$ inactivation and found in $38 \%$ of PGDSCre; $N f 2^{f l o x} f f l o x 2$ mice: Meningothelial tumors were originating from the arachnoid layer (arachnoid border cells) and fibroblastic tumors were emanating from the dura-mater (dural border cell layer) [12] (Fig. 4). In contrast, for Smo-related meningeal tumorigenesis, only meningothelial and no fibroblastic meningiomas were found in PGDSCre;SmoM2 mice, thus implying that, unlike PGDS-positive arachnoidal cells, PGDS-positive dura-mater cells are not vulnerable to Smo activation for meningothelial meningioma development (Fig. 4). All meningiomas found in PGDSCre;SmoM2 mice displayed histological features strikingly similar to human $S M O$-mutant meningiomas. To explore the postnatal developmental window for tumor development, we have previously reported a model with adCre injection in the subdural space of newborn mice and shown that $N f 2$ loss in very early postnatal meningeal cells was sufficient to induce meningioma formation (WHO Grade I meningiomas of different histological subtypes in $23 \%$ of mice) [12]. In contrast to $N f 2$-related tumorigenesis, the absence of meningioma in adCre;SmoM2 mice, corresponding to an early postnatal activation, and the selective finding of meningothelial (WHO Grade I) meningiomas at the skull base level of PGDSCre;SmoM2 mice suggest that embryonic activation of Smo in the arachnoidal meningeal layer is necessary for Smo-related meningioma tumorigenesis (Fig. 4). The temporal developmental window in which Smo mutation culminates in meningioma formation is more restrictive for Smo compared to $N f 2$ where the window for tumor initiation is wider, encompassing the embryonic and early postnatal periods [12]. The benign nature and unique spatial location of these meningiomas is also reflected by the slight in vitro proliferative advantage and selective sensitivity to Sonidegib of SmoM2-expressing arachnoidal cells of the skull base versus the convexity and by the presence of meningothelial proliferation restricted to the skull base level regardless of the injection site of adCre, respectively.

Taken together, these results indicate that Smo activation is necessary and sufficient to induce meningioma development at the skull base level in a restricted temporal window during the prenatal period starting at E12.5, strongly arguing in favor of the developmental nature of this subgroup of meningiomas and supporting the hypothesis that the developmental stage of arachnoidal cells determine their susceptibility to tumor-initiating alterations, This hypothesis and the predominance of human $S M O$-mutated meningiomas at the median skull base level are also sustained by the 

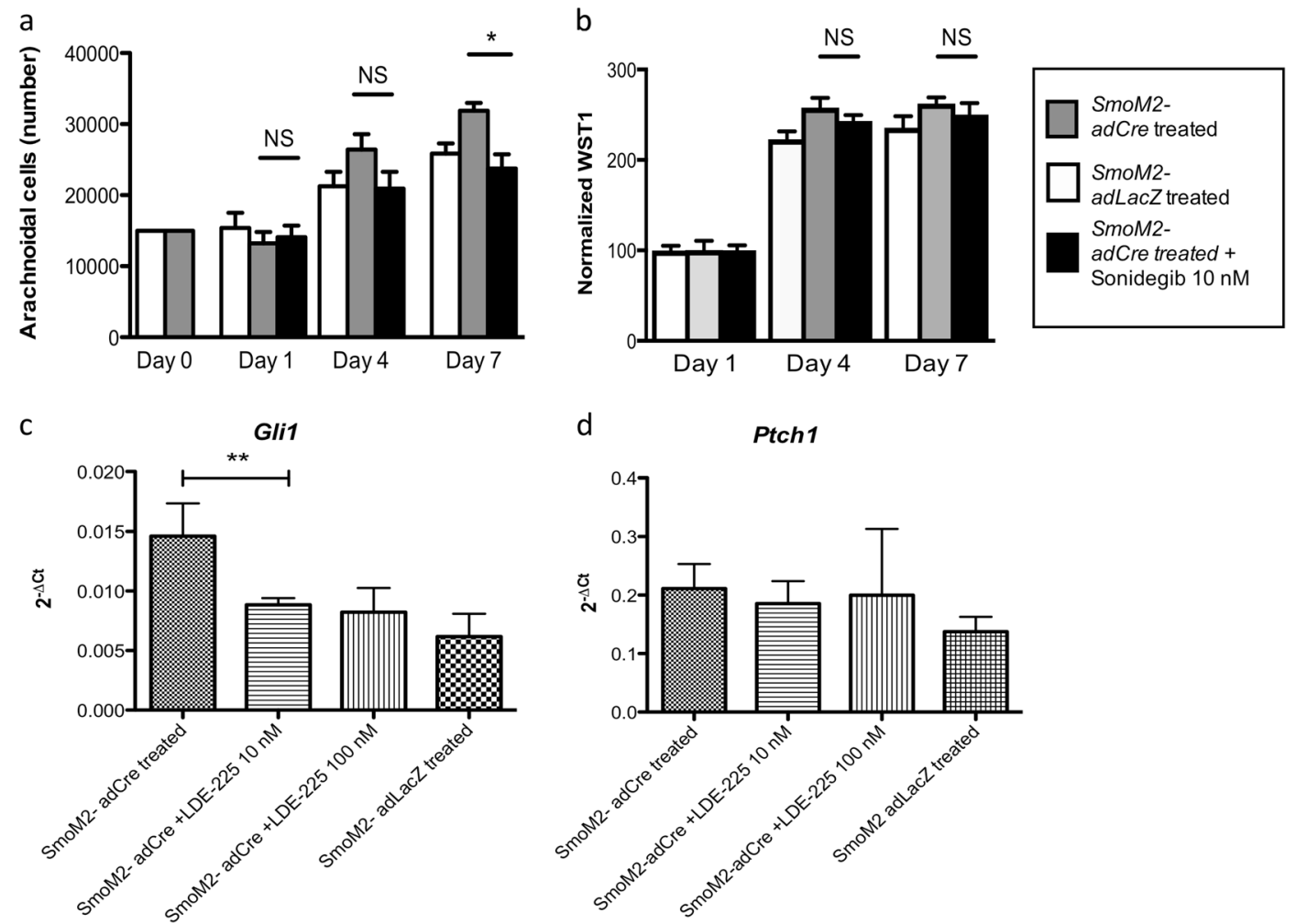

Fig. 3 Effect of Sonidegib (LDE-225) on proliferation and SHH pathway in skull base SmoM2-induced cells. Sonidegib at $10 \mathrm{nM}$ reversed the proliferation advantage of SmoM2-induced cells at day 7 when counting the cells and in WST1 assay (a, $p=0.02, t$-test and $\mathbf{b}$,

$p=0.41, t$-test), and Gli1 mRNA expression (c, $p=0.005, t$-test $)$ compared to vehicle (DMSO). No significant effect was found on Ptch1 mRNA levels (d, $p=0.71, t$-test). All experiments were performed in triplicate

fact that $\mathrm{SHH}$ is the main embryonic signaling pathway implicated in ventral craniofacial development by promoting rapid cell expansion of the facial mesenchyme, quickly followed by a transition to a terminally differentiated state of the connective tissue of the face and skull [16, 19, 20]. It has already been shown that targeted disruption of Smo in mice affect appropriate growth and patterning in the early facial primordial, while its constitutive activation (Wnt1Cre;SmoM2) produces hyperplasia of the facial processes and gross disorganization, with a failure of most craniofacial skeletogenesis [9]. Identically, in ameloblastoma, a locally destructive tumor occurring during tooth development, a relation between anatomical site and driver gene mutation has also been recently shown: Mutations in SMO are common in ameloblastomas arising from the maxilla whereas $B R A F$ mutations are predominant in tumors of the mandible [21]. This anatomical specificity for odontogenic development in the upper or lower dentition is likely to reflect distinctive developmental pathways based on spatial temporal and/or cell type-specific cues. Finally, similar to our approach and results, Ohli et al.[22] have observed in a Math1-CreER ${ }^{T 2} ; \mathrm{Smo}^{M 2}$ medulloblastoma mouse model that post-natally induced activation of $S m o^{M 2}$ lead to a different tumor burden compared to embryonically induced activation of $\mathrm{Smo}^{M 2}$. Their results were in line with the distribution of $\mathrm{SHH}$ medulloblastomas in human and indicate that the localization of $\mathrm{SHH}$ medulloblastomas was dependent on the time-restricted susceptibility of granule cell precursors in defined cerebellar compartments.

Curiously, no PGDSCre;SmoM2 mouse suffered from symptomatic meningioma before 15 months of age. This is likely due to slow, indolent tumor growth with no clinical impact until late in life, like in humans, where SMO-mutant meningiomas are also diagnosed later in life (mean age of 55 years) [13]. In a recent paper, an integrated model of the growth dynamics of benign meningiomas using radiocarbon retrospective birth dating was provided for the first time [23]. In their series, the mean lifetime of WHO grade I meningiomas was $22.1 \pm 6.5$ years (whereas atypical WHO grade II meningiomas originated $1.5 \pm 0.1$ years) prior to surgery. Assuming an additional period of time between the first initiating mutation in a single meningeal cell and the time a microscopic lesion can be measured, in addition to the fact that olfactory groove meningiomas (like most SMO-mutated meningiomas [13]) can remain silent for a long time despite a very large volume, then we should reconsider our concepts on the natural history of grade I 


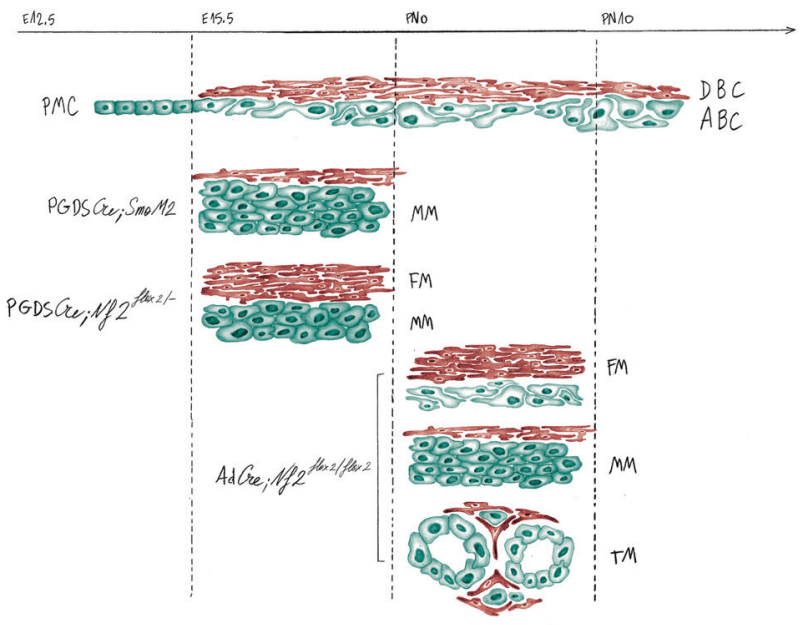

Fig. 4 Illustration of the mouse Grade I skull base meningiomas in relation with the meningeal cell of origin and the mutation of the Smo or $N f 2$ genes: A restricted developmental temporal and spatial window for Smo activation for meningioma vulnerability in comparison with $N f 2$ inactivation. In the normal meninges of skull base, primordial meningeal cells (PMC) (starting at E12.5) undergo lineage commitment to form both dural border cells (DBC) and arachnoid barrier cells $(\mathrm{ABC})$ at E15.5. Early mutation of Smo at the embryonic cell stage leads only to the development of meningothelial meningioma (MM) in PGDSCre;SmoM2 mice. In contrast, mutation of Smo at a later developmental stage, following early postnatal (PN1) adenoviral Cre injection (AdCre;SmoM2 mice), has no effect on tumorigenesis. For $N f 2$-related meningioma tumorigenesis, early $N f 2$ loss in embryonic primordial meningeal cells (PGDSCre; $N f 2^{\text {flox } 2 /-}$ mice) induces the development of fibroblastic meningiomas (FM) in the dura mater and meningothelial meningiomas (MM) in the arachnoid layer. All histological subtypes, including transitional (TM), were observed following early postnatal (PN1-10) Nf2 inactivation by adenoviral Cre injection (AdCre;Nf $2^{\text {flox } 2 / \text { flox } 2}$ mice)

meningiomas. In contrast, other developmental malignant tumors of the central nervous system, such as neuroblastomas, SMO-mutant or SHH-activated medulloblastomas, and malignant rhabdoid tumors generally occur during infancy or childhood [16, 24]. The restricted early developmental time window for Smarcbl loss to promote malignant rhabdoid tumor formation and vulnerability to additional $N f 2$ tumor suppressor gene mutations resulting in benign schwannoma formation later in life, provides another striking example of close interrelationship between timing of driving mutations, tumor tissue of origin, and clinical behavior [25].

From a prognostic and therapeutical point of view, despite their bland histologic appearance, we have shown that $S M O$ mutation is associated with poorer prognosis within the olfactory groove meningiomas subgroup with several patients showing serious clinical morbidity (blindness, pituitary gland dysfunction) and late recurrences [13]. These recurrences were observed despite optimal available treatments, suggesting that SMO inhibitors would be clinically useful in daily practice. SMO inhibitors are under clinical evaluation for SHH-mutated medulloblastoma [26] and routinely used in metastatic basal cell carcinoma [27]. Our study provides the first preclinical in vitro evidence of the efficiency of Sonidegib for the treatment of SMOmutant meningiomas. Sonidegib significantly inhibited both Shh pathway activation and growth in SmoM2 activated skull base arachnoidal cells at nanomolar concentrations. Thus, we provide a rationale for clinical trials evaluating SMO inhibitors in the treatment of refractory $S M O$-mutant meningiomas. A multicenter phase II study of Vismodegib is now recruiting patients with progressive or recurrent SMO-mutant meningiomas (NCT02523014, clinicaltrials.gov).

\section{Materials and methods}

\section{Mice}

Rosa26-lox-STOP-lox-SmoM2 mice (called SmoM2 mice in this paper) were purchased from the Jackson Laboratory. In this model, a conditional allele of SmoM2 with a C-terminal YFP tag is targeted into the ubiquitously expressed Rosa26 locus, after a lox-P flanked polyadenylation stop sequence cassette. In presence of Cre recombinase, SmoM2 gene is expressed and encodes for a mutant form of protein Smo previously identified in human basal cell carcinoma [28]. In this mutant allele, an activating mutation (W535L) in the seventh transmembrane domain results in ligandindependent constitutive activation of the Shh pathway in target tissue [14].

To induce postnatal SmoM2 activation in meningeal cells, we first performed injections in the sub-dural space of 3-4 $\mu \mathrm{L}$ of adenoviruses Ad5CMV-Cre (REF \#VVC-U of Iowa-5, University of Iowa, Viral Vector Core, Iowa City, IA, USA), called further adCre, in SmoM2 pups (postnatal day 2) as previously described (trans-orbitary (t.o.) to target the skull base and subdurally (s.d.) to target the meninges at the convexity) [15]. A control group of SmoM2 pups were injected with Ad5CMVntLacZ (REF \#VVC-U of Iowa-1917, University of Iowa, Viral Vector Core, Iowa City, IA, USA), called adLacZ, with the same methodology.

To induce prenatal activation of Shh in meningeal precursor cells, SmoM2 mice were bred with homozygous PGDSCre mice to obtain hemizygous PGDSCre;SmoM2 mice [12]. In this model, Cre recombinase is expressed selectively in PGDS-positive cells and is able to induce the expression of the SmoM2 gene. All animal care and experimentation reported herein were conducted in compliance with the guidelines and with the specific approval of the Institutional Animal Care and Use Committee of the French Department of Agriculture. 


\section{Histopathology and immunohistochemistry}

Mice were monitored closely and killed when seriously ill or at 15 months. To preserve the arachnoidal layer, the head was fixed in formalin in toto, decalcified, and sliced coronally before embedding in paraffin as previously described [29]. The terminology used for the description of the meningothelial lesions in the mouse models is based on the WHO classification of human tumors with adaptations [30]. The term "meningothelial hyperplasia" is not used, as in humans where it refers to the proliferation of reactive, normal arachnoidal cells. "Meningothelial proliferation" refers to very small (microscopic) lesions composed of meningothelial cells that represent early tumor formation. "Meningioma" refers to a larger meningothelial lesion, with features similar to a WHO grade I meningioma in humans. Immunohistochemical staining was performed on $5-\mu \mathrm{m}$ paraffin sections. For antigen retrieval, after pressure boiling in $1 \mathrm{mM}$ EDTA ( $\mathrm{pH}$ 8.0) for $30 \mathrm{~min}$, sections were blocked in $10 \%$ serum followed by incubation with primary antibody. Biotinylated secondary antibodies were used at 1:200 (Gli-1, ab151796, Abcam). Stainings were developed using Vectastain $\mathrm{ABC}$ system and $\mathrm{DAB}$ substrate kit (Vector Labs). Normal mouse arachnoid was used as positive control.

\section{Arachnoidal cells culture}

Primary arachnoidal cells cultures were established from SmoM2 mice. The arachnoidal tissue was surgically removed at the ventral surface of the brainstem (skull base arachnoid) and at the convexity (convexity arachnoid) after killing and plated in Petri dishes after 30 min digestion with Collagenase III (Wothington, Lakewood, NJ, USA). Arachnoidal cells were then cultured in Dulbecco's modified Eagle's medium (DMEM; Gibco/Invitrogen, Carlsbad, CA, USA) containing 10\% Fetal Calf Serum (FCS), insulin $0.05 \%$, Penicillin/streptomycin 1\%, and Epidermal Growth Factor $0.004 \%$. After 15 days of primary culture, cells were passaged in 12-well plates. Cells were infected after $48 \mathrm{~h}$ either with adCre (group called "SmoM2-adCre treated") or with adLacZ (control group, called "SmoM2-adLacZ treated") at $50 \mathrm{pfu} / \mathrm{cells}$ for $24 \mathrm{~h}$. Expression of YFP was verified using a fluorescence microscope (Nikkon, IHC software) to assess SmoM2-YFP expression.

\section{In vitro functional assays}

\section{Proliferation assay}

After infection, arachnoidal cells were passaged, seeded at a concentration of 15,000 cells $/ \mathrm{mL}$ in triplicate in 12-well plates, and counted manually using a Malassez cell counting chamber 1, 4, and 7 days after plating. Arachnoidal cells were seeded at a concentration of 3000 cells $/ 100 \mu \mathrm{L}$ in triplicate in 96-well plates. WST1 assay was performed by adding 10\% WST1 reagent (Roche, Indianapolis, Indiana, USA) in the medium and measuring absorbance after $3 \mathrm{~h}$ using a Spectromax M4 for wavelengths $450-620 \mathrm{~nm}$.

\section{Senescence assay}

SA $\beta$-galactosidase staining was performed on infected arachnoidal cells [31].

\section{Apoptosis assay}

After infection, arachnoidal cells were passaged, stained according to a standardized Annexin V FITC-Propidium Iodide protocol (Thermo Fisher Scientific, Waltham, MA, USA) and sorted using flow cytometry analysis (FACSDiva, BD Biosciences, San Jose, CA, USA).

\section{RNA extraction and RT-qPCR}

After infection, cells were passaged and seeded in 12-well plates. RNA was extracted $48 \mathrm{~h}$ after passage using a Nucleospin RNA kit (Macherey-Nagel, Germany) and quantified using Nanodrop (Thermo Fisher, Villebon sur Yvette, France). RNA was retrotranscribed using the Maxima First Strand cDNA Synthesis Kit (Thermo Fischer). The cDNA obtained was used as a template for the determination of Glil and Ptchl mRNA expression by qPCR on a Lightcycler ${ }^{\circledR} 96$ (Roche, Bâle, Switzerland) using a Quantifast assay (Qiagen, Hilden, Germany). The $\Delta \mathrm{Ct}$ method was applied to normalize the mRNA levels using the expression of glucuronidase- $\beta$. The primer sets for qPCR were designed with the Roche design center (www. universalprobelibrary.com) (Supplementary Table S2).

\section{LDE-225 (Sonidegib) treatment}

Sonidegib, a selective inhibitor of SMO, was provided by Novartis (MTA 43219). Proliferation assays and Glil and Ptchl expression analyses were performed with Sonidegib at different concentrations (10 and $100 \mathrm{nM})$ in triplicate.

\section{Statistical analysis}

Student's $t$-tests were used in proliferation assays and mRNA level analyses. The distribution of categorical variables was compared with Fisher's exact test. All tests were two-sided and a $p$-value of $\leq 0.05$ was considered to be statistically significant. Statistical analyses were performed using Statview version 5.0 software (SAS Institute, Cary, NC, USA). 
Acknowledgements We are grateful to Marco Giovannini for critical reviewing of the manuscript. We are indebted to Marine Giry and Amithys Rahimian (Onconeurotek) for technical assistance, and Veronique Parietti and Martine Chopin for mouse handling.

Funding: This work was supported by a grant from the foundation ARC (PJA 20131200431), France. J.B. was funded by a grant from UM1 University, Montpellier, France.

Ethical approval: All procedures performed in studies involving animals were in accordance with the ethical standards of the institution at which the studies were conducted, and all applicable national guidelines for the care and use of animals were followed.

\section{Compliance with ethical standards}

Conflict of interest The authors declare that they have no conflict of interest.

\section{References}

1. Louis DN, Ohgaki H, Wiestler OD. WHO classification of tumours of the Central Nervous System, Revised.. Fourth Edition. Lyon: International Agency for Research On Cancer; 2016.

2. Rogers L, Barani I, Chamberlain M, Kaley TJ, McDermott M, Raizer J, et al. Meningiomas: knowledge base, treatment outcomes, and uncertainties. A RANO review. J Neurosurg. 2015;122:4-23.

3. Clark VE, Erson-Omay EZ, Serin A, Yin J, Cotney J, Ozduman $\mathrm{K}$, et al. Genomic analysis of non-NF2 meningiomas reveals mutations in TRAF7, KLF4, AKT1, and SMO. Science. 2013;339:1077-80.

4. Clark VE, Harmanc1 AS, Bai H, Youngblood MW, Lee TI, Baranoski JF, et al. Recurrent somatic mutations in POLR2A define a distinct subset of meningiomas. Nat Genet. 2016;48:1253-9.

5. Abedalthagafi M, Bi WL, Aizer AA, Merrill PH, Brewster R, Agarwalla PK, et al. Oncogenic PI3K mutations are as common as AKT1 and SMO mutations in meningioma. Neuro-Oncology. 2016;18:649-55.

6. Kros J, de Greve K, van Tilborg A, Hop W, Pieterman H, Avezaat $\mathrm{C}$, et al. NF2 status of meningiomas is associated with tumour localization and histology. J Pathol. 2001;194:367-72.

7. Reuss DE, Piro RM, Jones DTW, Simon M, Ketter R, Kool M, et al. Secretory meningiomas are defined by combined KLF4 K409Q and TRAF7 mutations. Acta Neuropathol. 2013;125:351-8.

8. Yuzawa S, Nishihara H, Yamaguchi S, Mohri H, Wang L, Kimura $\mathrm{T}$, et al. Clinical impact of targeted amplicon sequencing for meningioma as a practical clinical-sequencing system. Mod Pathol. 2016;29:708-16.

9. Jeong J, Mao J, Tenzen T, Kottmann AH, McMahon AP. Hedgehog signaling in the neural crest cells regulates the patterning and growth of facial primordia. Genes Dev. 2004;18:937-51.

10. Yoshida T, Vivatbutsiri P, Morriss-Kay G, Saga Y, Iseki S. Cell lineage in mammalian craniofacial mesenchyme. Mech Dev. 2008;125:797-808.

11. McBratney-Owen B, Iseki S, Bamforth SD, Olsen BR, MorrissKay GM. Development and tissue origins of the mammalian cranial base. Dev Biol. 2008;322:121-32.

12. Kalamarides M, Stemmer-Rachamimov AO, Niwa-Kawakita M, Chareyre F, Taranchon E, Han Z-Y, et al. Identification of a progenitor cell of origin capable of generating diverse meningioma histological subtypes. Oncogene. 2011;30:2333-44.
13. Boetto J, Bielle F, Sanson M, Peyre M, Kalamarides M. SMO mutation status defines a distinct and frequent molecular subgroup in olfactory groove meningiomas. Neuro-Oncology. 2017;19:345-51. https://doi.org/10.1093/neuonc/now276

14. Mao J, Ligon KL, Rakhlin EY, Thayer SP, Bronson RT, Rowitch $\mathrm{D}$, et al. A novel somatic mouse model to survey tumorigenic potential applied to the Hedgehog pathway. Cancer Res. 2006;66:10171-8

15. Kalamarides M, Niwa-Kawakita M, Leblois H, Abramowski V, Perricaudet M, Janin A, et al. Nf2 gene inactivation in arachnoidal cells is rate-limiting for meningioma development in the mouse. Genes Dev. 2002;16:1060-5.

16. Marshall GM, Carter DR, Cheung BB, Liu T, Mateos MK, Meyerowitz JG, et al. The prenatal origins of cancer. Nat Rev Cancer. 2014;14:277-89.

17. Ellison DW, Dalton J, Kocak M, Nicholson SL, Fraga C, Neale G, et al. Medulloblastoma: clinicopathological correlates of $\mathrm{SHH}$, WNT, and non-SHH/WNT molecular subgroups. Acta Neuropathol. 2011;121:381-96.

18. Varjosalo M, Taipale J. Hedgehog: functions and mechanisms. Genes Dev. 2008;22:2454-72.

19. Xavier GM, Seppala M, Barrell W, Birjandi AA, Geoghegan F, Cobourne MT. Hedgehog receptor function during craniofacial development. Dev Biol. 2016;415:198-215.

20. Eberhart JK, Swartz ME, Crump JG, Kimmel CB. Early Hedgehog signaling from neural to oral epithelium organizes anterior craniofacial development. Dev Camb Engl. 2006;133:1069-77.

21. Sweeney RT, McClary AC, Myers BR, Biscocho J, Neahring L, Kwei KA, et al. Identification of recurrent SMO and BRAF mutations in ameloblastomas. Nat Genet. 2014;46:722-5.

22. Ohli J, Neumann JE, Grammel D, Schüller U. Localization of SHH medulloblastoma in mice depends on the age at its initiation. Acta Neuropathol. 2015;130:307-9.

23. Huttner HB, Bergmann O, Salehpour M, El Cheikh R, Nakamura $\mathrm{M}$, Tortora $\mathrm{A}$, et al. Meningioma growth dynamics assessed by radiocarbon retrospective birth dating. EBioMedicine. 2017;27:176-81.

24. Kool M, Jones DTW, Jäger N, Northcott PA, Pugh TJ, Hovestadt V, ICGC PedBrain Tumor Project. et al. Genome sequencing of SHH medulloblastoma predicts genotype-related response to smoothened inhibition. Cancer Cell. 2014;25:393-405.

25. Vitte J, Gao F, Coppola G, Judkins AR, Giovannini M. Timing of Smarcb1 and Nf2 inactivation determines schwannoma versus rhabdoid tumor development. Nat Commun. 2017;8:300.

26. Samkari A, White J, Packer R. SHH inhibitors for the treatment of medulloblastoma. Expert Rev Neurother. 2015;15:763-70.

27. Migden MR, Guminski A, Gutzmer R, Dirix L, Lewis KD, Combemale $\mathrm{P}$, et al. Treatment with two different doses of sonidegib in patients with locally advanced or metastatic basal cell carcinoma (BOLT): a multicentre, randomised, double-blind phase 2 trial. Lancet Oncol. 2015;16:716-28.

28. Xie J, Murone M, Luoh SM, Ryan A, Gu Q, Zhang C, et al. Activating smoothened mutations in sporadic basal-cell carcinoma. Nature. 1998;391:90-92.

29. Giovannini M, Robanus-Maandag E, van der Valk M, NiwaKawakita M, Abramowski V, Goutebroze L, et al. Conditional biallelic Nf2 mutation in the mouse promotes manifestations of human neurofibromatosis type 2. Genes Dev. 2000;14:1617-30.

30. Peyre M, Stemmer-Rachamimov A, Clermont-Taranchon E, Quentin S, El-Taraya N, Walczak C, et al. Meningioma progression in mice triggered by $\mathrm{Nf} 2$ and Cdkn2ab inactivation. Oncogene. 2013;32:4264-72.

31. Le Roux I, Konge J, Le Cam L, Flamant P, Tajbakhsh S. Numb is required to prevent $\mathrm{p} 53$-dependent senescence following skeletal muscle injury. Nat Commun. 2015;6:8528. 